\title{
Dinámica multitemporal de las coberturas y el espejo de agua en la laguna de Fúquene
}

\author{
Ivón Maritza Castillo, Miguel Ángel Rodríguez*
}

Universidad Jorge Tadeo Lozano, Facultad de Ciencias Naturales e Ingeniería, Departamento de Ciencias Biológicas y Ambientales, maestría en Ciencias Ambientales, carrera 4 n.`22-61, módulo 16, oficina 309, Bogotá, Colombia.

* Autor para correspondencia: miguel.rodrigueza@utadeo.edu.co

\section{RESUMEN}

La laguna de Fúquene, ubicada en los valles de Ubaté y Chiquinquirá, es uno de los ecosistemas acuáticos más importantes del altiplano cundiboyacense, en Colombia. Desde hace varias décadas, la población circundante a la laguna se ha beneficiado de los servicios que ofrece este ecosistema, tales como la provisión de agua para la agricultura y los acueductos locales, pesca, turismo y transporte. Sin embargo, anteriores procesos de desecación y eutrofización de sus aguas, debidas a la carga orgánica y de nutrientes vertida de manera descontrolada sobre este cuerpo de agua, han ocasionado fuertes impactos ambientales sobre este ecosistema. Una de las principales evidencias del disturbio antrópico ha sido la progresiva reducción del espejo de agua a favor del crecimiento de diferentes tipos de coberturas vegetales acuáticas. Con el fin de analizar la dinámica de estas coberturas vegetales en el tiempo y en el espacio se emplearon herramientas de sensorización remota y sistemas de información geográfica. Mediante el análisis multitemporal de imágenes de satélite obtenidas en el periodo comprendido entre 1984 y 2003 se determinó que el área del espejo de agua presentó una disminución del 78.7 \%. Espacialmente se estableció que la aparición y expansión de la vegetación acuática se ha dado desde las orillas norte y sur de la laguna. La disponibilidad restringida de imágenes de satélite no permitió establecer la tendencia actual de las áreas del espejo de agua y de las coberturas vegetales. La aplicación de herramientas de sensorización remota y sistemas de información geográfica permitió una cuantificación bastante precisa de los cambios espaciales y temporales que han presentado las coberturas de la laguna a lo largo del periodo de tiempo considerado, aun cuando la desecación de la laguna y la pérdida del espejo de agua es un fenómeno plenamente comprendido para la laguna de Fúquene.

Citation: Castillo, I. M. \& Rodríguez, M. A. (2017). Dinámica multitemporal de las coberturas y el espejo de agua en la laguna de Fúquene. Mutis 7(1), 20-33, doi: http://dx.doi.org/10.21789/22561498.1183

Received: Septiembre 20, 2016. Accepted: Diciembre 12, 2016. Published on line: Mayo 1, 2017.

Copyright: $\odot 2017$ Castillo, I. M. \& Rodríguez, M. A. This is an open-access article, which permits unrestricted use, distributions and reproduction in any medium, provided the original author and source are credited.

Competing Interests: The authors have no conflict of interest.
Palabras clave: disturbio antrópico, eutrofización, impacto ambiental, sensorización remota, sistemas de información geográfica.

\section{Multitemporal cover dinamics in the Fúquene lagoon}

\section{ABSTRACT}

The Fúquene Lagoon, located in the valleys of Ubate and Chiquinquirá, is one of the most important 
aquatic ecosystems of the Altipano Cundiboyacense in Colombia. For several decades, the population surrounding the lagoon has benefited from the services this ecosystem provides such as water supply for agriculture and local communities, fisheries, tourism and transport. However, previous processes of desiccation and eutrophication of its waters, due to organic matter and nutrients discharged out of control on this body of water, have caused serious environmental impacts on the ecosystem. One of the main evidences of this anthropic disturbance has been the progressive reduction of the water surface in favor of the growth of different types of aquatic vegetation cover. In order to analyze the dynamics of these plant covers in time and space, this work was proposed in which tools from remote sensing and geographic information systems were employed. Through the multi-temporal analysis of satellite images for the period between 1984 and 2003 it was determined that the area of the water surface showed a decrease of $78.7 \%$. Spatially it was established that the emergence and spread of aquatic vegetation has ocurred from the north and south shores of the lake. The restricted availability of satellite images limited the possibility to establish the trend of the areas of water surface and vegetation cover. The application of remote sensing and geographic information systems allowed a fairly precise quantification of spatial and temporal changes along the period considered, despite the draining of the lagoon and loss water mirror is a fully understood phenomenon.

Keywords: anthropic disturbance; eutrophication; environmental impact; remote sensing; geographic information systems

\section{INTRODUCCIÓN}

Hoy en día la pérdida y degradación de ecosistemas acuáticos, así como la disminución de la calidad del recurso hídrico, constituyen una de las mayores preocupaciones ambientales a nivel mundial (NavarroOrtega et al., 2015). La presión antrópica sobre este tipo de ecosistemas, debida a sectores como el industrial o el agrícola, ha ocasionado problemas de tipo ambiental, entre los que se incluyen procesos de contaminación a diversas escalas, pérdida de hábitats de flora y fauna y afectación de los servicios ecosistémicos ofrecidos (e. g., Camargo y Alonso, 2006; Alberti et al., 2007; Woodward et al., 2012; Omar, Zaghloul, Abdel-Khalek \& Abo-Hegab, 2013). Colombia no ha sido ajena a esta situación: a lo largo de su territorio se encuentran ecosistemas acuáticos que presentan esta problemática en mayor o menor grado (Espitia-Contreras, 2013).

La laguna de Fúquene, ubicada en los valles de Ubaté y Chiquinquirá, ha sido uno de los ecosistemas acuáticos más fuertemente sometidos a diferentes procesos de disturbio antrópico. El principal de ellos es la ganadería que se adelanta en buena parte de la región circundante a la laguna, así como la agricultura desarrollada en las zonas de ladera, mayoritariamente con cultivos de papa. El uso indiscriminado de fertilizantes en estas zonas ha ocasionado procesos de eutrofización que favorecen la proliferación de coberturas vegetales acuáticas y que a su vez constituyen un factor determinante en la desecación de la laguna. Este tipo de procesos de contaminación se constituyen en amenazas locales a la dinámica de los ecosistemas acuáticos (Khan et al., 2014).

De igual manera, los vertimientos inadecuados de aguas residuales por parte de las poblaciones aledañas y de las industrias presentes en la zona también han contribuido a agravar la problemática que hoy exhibe esta laguna. La consecuencia directa y evidente de estas afectaciones ha sido la disminución del espejo de agua, pero también genera incertidumbre el efecto de estas afectaciones sobre la calidad del recurso hídrico del que se abastecen las poblaciones ubicadas aguas abajo (Pernett, 2006).

La integración de la sensorización remota y de los sistemas de información geográfica (SIG) han permitido el monitoreo y la evaluación de cambios espaciales y temporales de las coberturas en diferentes lugares del mundo (e. g., Sexton, Urban, Donohue y Song, 2013; Were, Dick, \& Singh, 2013; Pathirana, Perera y Sanaa, 2015). Hoy en día, la disponibilidad de imágenes de satélite de alta resolución, tanto temporal como espacial, permite analizar la variabilidad del agua almacenada en un determinado ecosistema en relación con aspectos clave como la susceptibilidad a inundaciones o la presencia de algún tipo de disturbio (Sawaya et al., 2003). Por medio de la información contenida en las imágenes de satélite, los SIG permiten cuantificar de manera confiable el área de la superficie del cuerpo 
de agua, elevación, pendiente y cambio temporal, variables que posibilitan analizar la variabilidad de los cuerpos de agua (Alsdorf, Rodríguez \& Lettenmaier, 2007).

La sensorización remota se ha convertido en una técnica alternativa útil y eficiente para monitorear y evaluar la dinámica permanente a que se ven expuestos los cuerpos de agua en diferentes lugares del mundo. La utilización de este método se ha visto potenciado por la creciente disponibilidad de imágenes satelitales multitemporales con resoluciones moderadas a altas durante las últimas tres décadas (Bastawesy, Khalaf, \& Arafat, 2008).

El comportamiento multitemporal de diversos cuerpos de agua a lo largo del mundo ha sido estudiado aplicando la sensorización y los SIG. HernándezCornejo, Koedam, Ruiz-Luna, Troell y DahdouhGuebas (2005) evaluaron los cambios en las áreas de manglar en el complejo lagunar Navachiste-San Ignacio-Macapule, en el estado de Sinaloa (México). Los cambios multitemporales del lago Uluabat (Turquía) y sus áreas circundantes fueron estudiadas por Aksoy \& Özsoy (2002), también utilizando la integración de la sensorización remota y los SIG. Alexandridis, Takavakoglou, Crisman, \& Zalidis (2007) plantearon como objetivo de su estudio demostrar cómo la sensorización remota, junto con los SIG, podían contribuir a la selección de una condición de referencia sostenible para el lago Koronia de Grecia.

En Grecia, Papastergiadou, Retalis, Apostolakis \& Georgiadis (2008) presentaron un estudio de caso en el que aplicaron la integración de estas herramientas para la clasificación y el monitoreo de los cambios espaciales y temporales de un humedal natural del lago Cheimaditida, ubicado en el norte de Grecia. Giardino, Bresciani, Villa \& Martinelli (2010) estudiaron el lago Trasimeno, en Italia, con el objetivo de mejorar el conocimiento ambiental y las condiciones de referencia de ese cuerpo de agua. Dicho estudio no solamente incluyó el análisis multitemporal y espacial de las coberturas, sino también la calidad del agua.

A escala regional se han utilizado herramientas de sensorización remota y SIG para analizar el comportamiento de diferentes cuerpos de agua. En relación con lagunas, Rondón y Vergara (2012) estudiaron espacial y temporalmente el comportamiento histórico de las lagunas Sayhuacocha, Raurac, Ampatococha y Tutayoc, ubicadas en el departamento de Huancavelica, en Perú. En Colombia, Parra \& Hernández (2010) identificaron y delimitaron los humedales lénticos del valle alto del río Cauca mediante el procesamiento digital de imágenes de satélite.

En relación con la laguna de Fúquene, se han estudiado aspectos relativos a su flora, fauna, conservación y problemática ambiental. Sin embargo, estudios relacionados con el uso de sensorización remota y SIG son pocos; entre ellos se encuentran el elaborado por Bustamante (2010), quien desarrolló un modelo de autómatas celulares para simular la invasión del buchón (Eichornia crassipes) durante el periodo 19502030. A partir de ocho fotografías aéreas tomadas entre los años 1950 y 2007 se extrajo la información de base requerida para la calibración del modelo.

Dada la problemática actual de la laguna de Fúquene se hace necesario realizar un análisis cuantitativo de la dinámica del espejo de agua y de las coberturas acuáticas presentes, con el fin de establecer una línea de base objetiva que permita conocer no solo los cambios a que se ha visto sometida la laguna, sino los posibles escenarios a que se vería abocada de no corregirse la situación actual. Es así como el objetivo del presente trabajo fue determinar la dinámica de las coberturas vegetales acuáticas y del espejo de agua de la laguna de Fúquene mediante el análisis multitemporal de imágenes de satélite durante el periodo comprendido entre 1984 y 2003.

\section{MATERIALES Y MÉTODOS}

\section{Área de estudio}

La cuenca de la laguna de Fúquene se encuentra en la sabana de los valles de Ubaté y Chiquinquirá, a una altura de 2543 m.s. n. m., entre los $5^{\circ} 25^{\prime}$ y $5^{\circ} 30^{\prime}$ de latitud norte y $73^{\circ} 43^{\prime}$ y $73^{\circ} 47^{\prime}$ de longitud oeste (Pernett, 2006); posee un área de $1752 \mathrm{~km}^{2}$, que forma parte del sistema hídrico integrado por los ríos Ubaté, Sutatausa, Cucunubá, Lenguazaque, Honda y Monroy; todos ellos desembocan en la laguna de Fúquene (Rodríguez-Briceño, 2012).

La zona se caracteriza por tener dos temporadas secas y dos húmedas, que se alternan. Las temporadas secas 
ocurren entre los meses de diciembre a febrero y de junio a agosto, mientras que las temporadas húmedas se presentan durante los meses de marzo a mayo y de septiembre a noviembre. La precipitación promedio anual es de $850 \mathrm{~mm}$, mientras que la temperatura mensual promedio varía en un rango de 12 a $13.2{ }^{\circ} \mathrm{C}$ (Pernett, 2006).

\section{Cuantificación del espejo de agua y coberturas vegetales}

La cuantificación del área del espejo de agua y de las coberturas vegetales acuáticas asociadas a la laguna de Fúquene se realizó mediante la interpretación de imágenes de los satélites Landsat 5 y Landsat 7 tomadas entre los años 1984 y 2003. Esas imágenes se descargaron gratuitamente de la página del Servicio Geológico de los Estados Unidos (USGS, por sus siglas en inglés). Cada imagen satelital está compuesta por un conjunto de archivos, cada uno de los cuales contiene la información registrada en cada una de las bandas espectrales. Las imágenes descargadas están radiométrica y geométricamente corregidas, y georreferenciadas con el sistema de coordenadas geográficas WGS84 (World Geodetic System 84).

Las imágenes de las bandas individuales se usaron para realizar una composición a falso color usando la combinación RGB 453, procedimiento que se llevó a cabo usando el software GRASS 6.3 (GRASS Development Team, 2012), por medio de la función r.composite. El uso de esta combinación de bandas ha demostrado ser útil para discriminar diferentes tipos de vegetación y espejos de agua (Tarasenko, 2013), en especial, porque las bandas 4 y 5 capturan la reflectancia en el infrarrojo cercano, y en este segmento del espectro las plantas tienen una alta reflectancia, lo que facilita su identificación y clasificación.

La interpretación se realizó con posterioridad a la proyección de las imágenes al datum oficial de coordenadas planas (Gauss-Krüger) para la franja central de Colombia: Magna-Sirgas/Colombia Bogota zone, identificada con el código EPSG (European Petroleum Survey Group) 3116.

Posteriormente se realizó un corte de las imágenes correspondiente al área de la laguna, que se definió con base en la construcción de un polígono que delimitaba el área a partir de la imagen más antigua (diciembre de 1984). Los recortes de las imágenes se interpretaron usando como principales criterios el color y el patrón espacial de los distintos tipos de coberturas presentes en cada imagen, fraccionando el espacio en diferentes clases caracterizadas por su homogeneidad interna y distintas de áreas adyacentes (Melo y Camacho, 2005). La delimitación de las coberturas se llevó a cabo mediante la segmentación del polígono inicial que se definió para delimitar la laguna. Este proceso se realizó usando el software QGIS (QGIS Development Team, 2009). Después de la delimitación y clasificación de las distintas coberturas se determinó su área, procedimiento que se realizó con cada una de las once imágenes obtenidas.

Para la definición de las categorías correspondientes a la cobertura vegetal acuática se revisó la literatura existente relacionada con la vegetación presente en la laguna y se usó como guía el trabajo realizado por Vidal y Pérez (2007). Se definieron las siguientes categorías para clasificar las coberturas en el área de la laguna de Fúquene:

- Espejo de agua: superficie donde se aprecia la presencia de agua sin presencia relevante de vegetación. En esta categoría los colores varían del azul oscuro al negro, y los bordes del polígono tienden a ser irregulares.

- Vegetación emergente de ribera: vegetación que se encuentra en los bordes de la laguna. En el caso de la laguna de Fúquene, esta vegetación está compuesta principalmente por varias especies de juncos (Schoenoplectus californicus y Juncus effusus) y la enea o totora (Typha angustifolia). En la combinación de bandas empleada, esta vegetación presenta un color verde claro.

- Vegetación acuática flotante: vegetación que se encuentra dentro de la laguna. En la laguna de Fúquene las más abundantes son plantas vasculares: helecho de agua (Azolla filiculoides), lenteja de agua (Lemna minuscula), buchón de agua (Limnobium laevigatum) y la elodea (Egeria densa). Todas ellas se distinguen por una coloración violeta.

- Praderas emergentes: constituidas por especies vegetales como el clavito de agua (Ludwigia peploides), la gualola (Polygonum segetum) y el barbasco (Polygonum punctatum) y algunos juncos como la totora (Typha angustifolia). En la combinación de bandas se distinguen por colores rosados. 
- Áreas cultivadas: áreas dedicadas principalmente al pastoreo de ganado, donde se destaca la presencia de pasto kikuyo (Pennisetum clandestinum). Estas áreas tienen formas regulares con bordes rectos, y con la combinación de bandas empleada se observan de color verde claro.

\section{Determinación de la variación del espejo de agua y coberturas vegetales}

Una vez determinadas las áreas de las coberturas vegetales y el espejo de agua de la laguna en diferentes fechas, se procedió a construir una serie de tiempo para cada una de ellas; adicionalmente, se determinó la variación porcentual de cada cobertura en la imagen más reciente (febrero de 2003), tomando como referencia la cobertura en la más antigua (diciembre de 1984).

A partir de las áreas ocupadas por el espejo de agua y las coberturas acuáticas vegetales se establecieron las posibles asociaciones entre las coberturas discriminadas a lo largo del tiempo, para lo cual se calcularon las correlaciones lineales de Rho-Spearman entre pares de coberturas. Con el fin de profundizar en las variaciones que ha presentado el espejo de agua se realizó una regresión lineal entre el área del espejo de agua y el tiempo. Al calibrar el modelo de regresión lineal se obtuvieron los parámetros de intercepto y pendiente, siendo la pendiente el valor que se analizaría. Estos procedimientos se realizaron usando el lenguaje de programación estadístico R (R Core Team, 2014).

\section{RESULTADOS Y DISCUSIÓN}

\section{Cuantificación del espejo de agua y coberturas vegetales}

La cuantificación del espejo de agua y de las coberturas vegetales acuáticas presentes en el momento de la toma de cada imagen satelital se encuentran presentadas en la tabla. En el momento de la captura de la primera imagen satelital considerada en el presente estudio solamente se determinó la presencia notable de vegetación emergente de ribera, mientras que el área ocupada por las praderas emergentes podía considerarse como marginal. Para esa fecha, el espejo de agua dominaba la mayor parte del área de la laguna, representando el $62.3 \%$ del área del polígono estudiado.

Tabla. Distribución de las áreas $\left(\mathrm{km}^{2}\right)$ ocupadas por el espejo de agua y las coberturas vegetales acuáticas de la laguna de Fúquene a partir de la interpretación de las imágenes de satélite consideradas.

\begin{tabular}{|c|c|c|c|c|c|c|}
\hline $\begin{array}{c}\text { Fecha de } \\
\text { imagen }\end{array}$ & $\begin{array}{c}\text { Acuática } \\
\text { flotante }\end{array}$ & $\begin{array}{c}\text { Áreas } \\
\text { cultivadas }\end{array}$ & $\begin{array}{c}\text { Emergente } \\
\text { de ribera }\end{array}$ & $\begin{array}{c}\text { Espejo } \\
\text { de agua }\end{array}$ & Isla & $\begin{array}{c}\text { Praderas } \\
\text { emergentes }\end{array}$ \\
\hline Dic. 1984 & 0.0 & 0.0 & 11.3 & 19.8 & 0.1 & 0.5 \\
\hline Jul. 1985 & 0.0 & 0.0 & 10.9 & 19.7 & 0.1 & 1.1 \\
\hline Mar. 1987 & 0.0 & 0.0 & 11.3 & 19.1 & 0.1 & 1.2 \\
\hline Dic. 1987 & 0.0 & 1.4 & 10.1 & 18.8 & 0.1 & 1.4 \\
\hline Dic. 1989 & 0.0 & 1.3 & 11.1 & 17.4 & 0.2 & 1.8 \\
\hline Mar. 1991 & 0.0 & 1.4 & 10.0 & 18.1 & 0.1 & 2.2 \\
\hline Oct. 1991 & 0.0 & 1.8 & 9.1 & 18.2 & 0.1 & 2.5 \\
\hline Feb. 2000 & 7.5 & 1.4 & 9.4 & 7.3 & 0.1 & 6.1 \\
\hline Ago. 2002 & 7.0 & 1.6 & 9.7 & 6.2 & 0.1 & 7.1 \\
\hline Nov. 2003 & 9.7 & 1.6 & 9.5 & 3.9 & 0.1 & 7.0 \\
\hline Dic. 2003 & 8.2 & 1.7 & 9.1 & 4.2 & 0.1 & 8.4 \\
\hline
\end{tabular}


En julio de 1985 la composición de espejo de agua y de las coberturas vegetales acuáticas en la laguna de Fúquene era similar a la de diciembre de 1984. Se apreció una leve disminución del área de vegetación emergente de ribera, que fue ocupada por la cobertura de praderas emergentes. Para la fecha de la siguiente imagen satelital considerada (marzo de 1987), el área ocupada por la vegetación emergente de ribera volvió a los niveles observados en 1984, mientras que la vegetación de praderas emergentes presentó un leve aumento en el área ocupada. En esa fecha ya se empezó a observar de manera más clara la reducción del área del espejo de agua.

En diciembre de 1987, la interpretación de la imagen satelital identificó por primera vez la presencia de áreas cultivadas, mientras que se confirmó la tendencia observada en la imagen de la fecha anterior de reducción del área del espejo de agua. El área ocupada por la cobertura de praderas emergentes continuó su progresivo aumento, mientras que la de vegetación emergente de ribera continuó exhibiendo un comportamiento fluctuante en relación con las imágenes de fechas anteriores.

Dos años después, en 1989, el área del espejo de agua continuaba su descenso progresivo, mientras que las áreas de vegetación emergente de ribera y de praderas emergentes presentaban crecimiento en las áreas ocupadas. El área de cultivos permaneció constante en esa fecha, comparada con la anterior imagen analizada. En marzo de 1991 el área de cultivos continuó presentando un área similar a la de las imágenes anteriores, mientras que el área de praderas emergentes continuó incrementando el área ocupada en la laguna. Para esa fecha, la tendencia de disminución del área del espejo de agua se revirtió y se observó un incremento de $0.7 \mathrm{~km}^{2}$ con respecto al área discriminada en diciembre de 1989.

En octubre de 1991 se confirmó la recuperación del espejo de agua, al encontrarse un valor muy similar al de marzo del mismo año. Sin embargo, las áreas ocupadas por cultivos y praderas emergentes exhibieron aumentos de 0.4 y $0.3 \mathrm{~km}^{2}$, respectivamente. El área de la vegetación emergente de ribera presentó una disminución de 0.9 km².

La siguiente imagen (4 de febrero de 2000) presenta una diferencia de tiempo de aproximadamente nueve años con respecto a la de octubre de 1991, por lo que se observan cambios drásticos en la composición de las áreas del espejo de agua y las coberturas vegetales acuáticas discriminadas. Es así como el área ocupada por la vegetación emergente de ribera es la mayor de todas las analizadas, incluso por encima que la ocupada por el espejo de agua. Para esa fecha, el área del espejo de agua se redujo en $10.9 \mathrm{~km}^{2}$, en comparación con la última imagen de 1991. De acuerdo con el análisis de las áreas, la cobertura de vegetación acuática flotante, que apareció por primera vez, fue la mayor responsable de la disminución del área del espejo de agua de la laguna para esa fecha.

En 2002 persistía la tendencia de disminución del área del espejo de agua a favor del aumento de todas las coberturas vegetales acuáticas consideradas, con excepción de la vegetación acuática flotante, que presentó una ligera disminución del área reportada en la imagen anterior. En noviembre de 2003, el área ocupada por el espejo de agua presentó una disminución de $2.3 \mathrm{~km}^{2}$ con respecto a la imagen anterior, área que fue ocupada principalmente por la vegetación acuática flotante, que registró un incremento de $2.7 \mathrm{~km}^{2}$. Las demás coberturas presentaron áreas similares a las discriminadas en la imagen de la fecha anterior.

La última imagen analizada, tomada en diciembre de 2003, muestra algunos cambios en las áreas de las coberturas consideradas. El área del espejo de agua presentó un ligero incremento de $0.3 \mathrm{~km}^{2}$, mientras que la vegetación acuática flotante y la emergente de ribera se redujeron en 1.5 y $0.4 \mathrm{~km}^{2}$, respectivamente. El área ocupada por cultivos permaneció prácticamente invariable con respecto a la información obtenida en la imagen anterior, mientras que la vegetación de praderas emergentes se incrementó en 1.4 km².

\section{Variación multitemporal del espejo de agua y coberturas vegetales}

A partir de la interpretación de la primera imagen satelital, tomada en diciembre de 1984, se determinó que el área del espejo de agua era de $19.8 \mathrm{~km}^{2}$, lo cual correspondía al $62.5 \%$ del área total de la laguna. En febrero del año 2003 el área del espejo de agua fue de $4.2 \mathrm{~km}^{2}$, lo cual representa una disminución del $78.7 \%$ del área libre de vegetación con respecto a lo 
determinado para el año 1984. Esto significa que 15.6 $\mathrm{km}^{2}$ de espejo de agua fueron cubiertos por vegetación en el periodo comprendido entre 1984 y 2003. En la figura 1 se observa cómo la disminución del espejo de agua ha estado asociada a un incremento de las coberturas correspondientes a praderas emergentes y vegetación acuática flotante.

En la secuencia de las interpretaciones (figura 1) se puede observar que los mayores cambios ocurrieron durante la década de los noventa. En la imagen tomada en febrero del 2000 se aprecia una extensa área de la laguna ocupada por vegetación acuática flotante y praderas emergentes, lo cual no se observaba con tanta intensidad en la imagen de octubre de 1991. Desde el punto de vista espacial, se observa que la aparición y expansión de la vegetación acuática se ha dado desde las orillas norte y sur de la laguna. Esto coincide, al norte, con el nacimiento del río Suárez o Saravita, que es el principal desagüe natural de la laguna. Al sur la expansión de la vegetación coincide con la desembocadura del principal afluente de la laguna, el río Ubaté, y con la construcción de varios canales que sirven para drenar hacia la laguna los excesos de aguas en las zonas agrícolas que la rodean.

En la interpretación de las imágenes se puede observar cómo el número de canales ha venido creciendo, especialmente al sur del cuerpo de agua. La construcción de estos canales seguramente ha contribuido a aumentar el flujo de nutrientes inorgánicos y residuos orgánicos (heces fecales animales) en la interface agua-sedimento hacia la laguna, lo cual mejoraría las condiciones para que se expanda la vegetación acuática e iría en detrimento del espejo de agua (CAR, 2000). El flujo de sedimentos también trae como consecuencia la colmatación de la laguna y la consecuente reducción de la profundidad. Esto se evidencia en la aparición y expansión de la vegetación denominada praderas emergentes, que se encuentra asociada con aguas someras (ClavijoAwazacko y Amarillo-Suárez, 2013).

Durante el periodo de estudio también se pudo observar la aparición y consolidación de áreas cultivadas, especialmente en las riberas de los ríos Suárez y Ubaté. En particular, en el borde del río Ubaté se observa que áreas originalmente cubiertas con vegetación emergente de ribera (juncos) han sido desecadas y se han convertido en áreas cultivadas, principalmente con pastos. En 2003, las áreas cultivadas correspondían al $5.4 \%$ de la laguna, es decir 173.08 hectáreas.

La figura 2 presenta la variación porcentual de las áreas de las coberturas consideradas en el presente trabajo, así como del espejo de agua. Desde 1984, la laguna se encuentra principalmente rodeada por juncos, cobertura que ha tenido una leve disminución (19.75\%), básicamente para dar paso a áreas cultivadas y a praderas emergentes. Las áreas cultivadas, que comenzaron a aparecer en las imágenes de satélite en diciembre de 1987, han presentado un comportamiento estable, con un porcentaje promedio de $4.8 \%$ del área del polígono de la laguna considerado en el presente trabajo. El área de praderas emergentes presentó un promedio de cobertura de $14.4 \%$, considerando todas las imágenes analizadas.

Esto confirma la reducción del espejo de agua, mientras que coberturas como las praderas emergentes o la vegetación acuática flotante han incrementado sus áreas de manera progresiva. El salto de información entre los años 1991 y 2000 hace que los aumentos o disminuciones de áreas sean visibles de manera explícita en la figura 3.

El análisis de correlaciones entre pares de coberturas mostró una correlación lineal positiva ( $P$ valor $<0.05$ ) entre la vegetación acuática flotante y de praderas emergentes, lo cual sugiere que las áreas ocupadas por estos dos tipos de coberturas han venido creciendo de manera conjunta, aunque cada una de ellas a su tasa particular. Adicionalmente, se presentaron correlaciones positivas ( $P$ valor $<0.05$ ) entre las áreas cultivadas y las coberturas de praderas emergentes y la vegetación acuática flotante.

En relación con el espejo de agua, se encontró una asociación lineal negativa ( $P$ valor $<0.05)$ de la vegetación acuática flotante $\mathrm{y}$ de praderas emergentes, lo cual indica que, durante el periodo de tiempo considerado, el área del espejo de agua disminuyó a favor del crecimiento del área ocupada para estas dos coberturas. No se evidenció mayor asociación entre la vegetación emergente de ribera y el espejo de agua.

Otra correlación lineal negativa observada tuvo lugar entre las áreas cultivadas y la vegetación emergente de ribera. Estas correlaciones son útiles 
Figura 1. Mapas de coberturas del área de la laguna de Fúquene durante el periodo comprendido entre 1984 y 2003 . Los mapas corresponden a cada una de las fechas en las que fue adquirida la imagen satelital
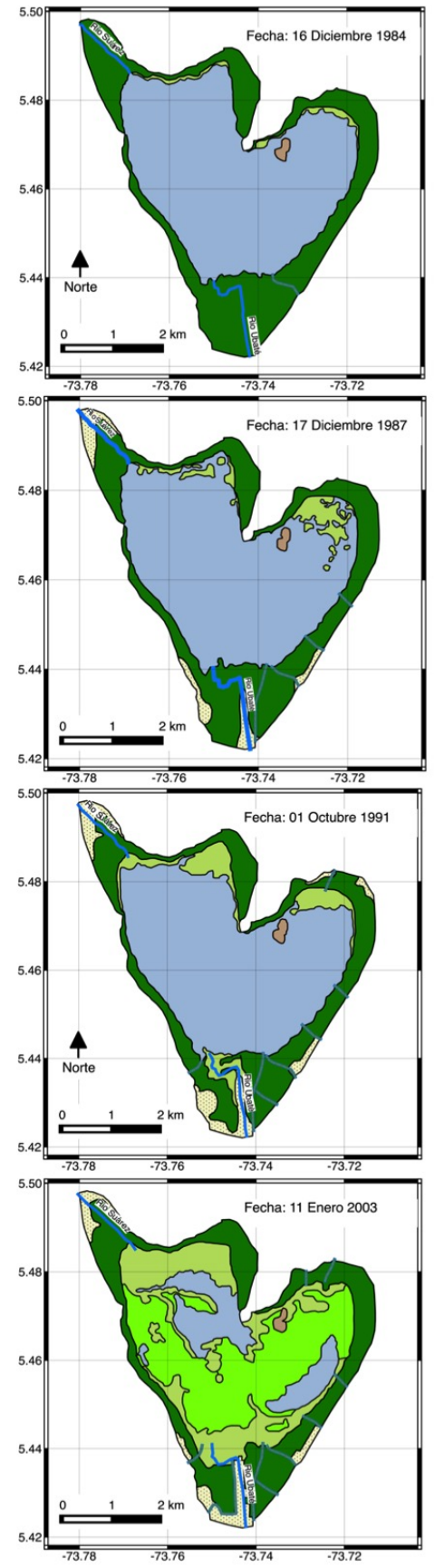
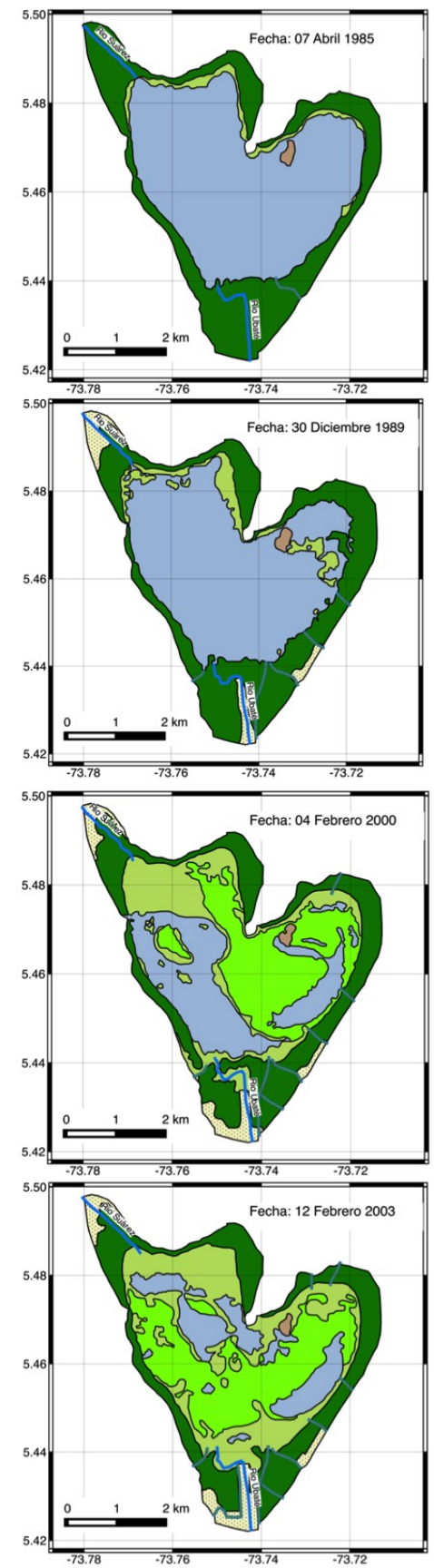
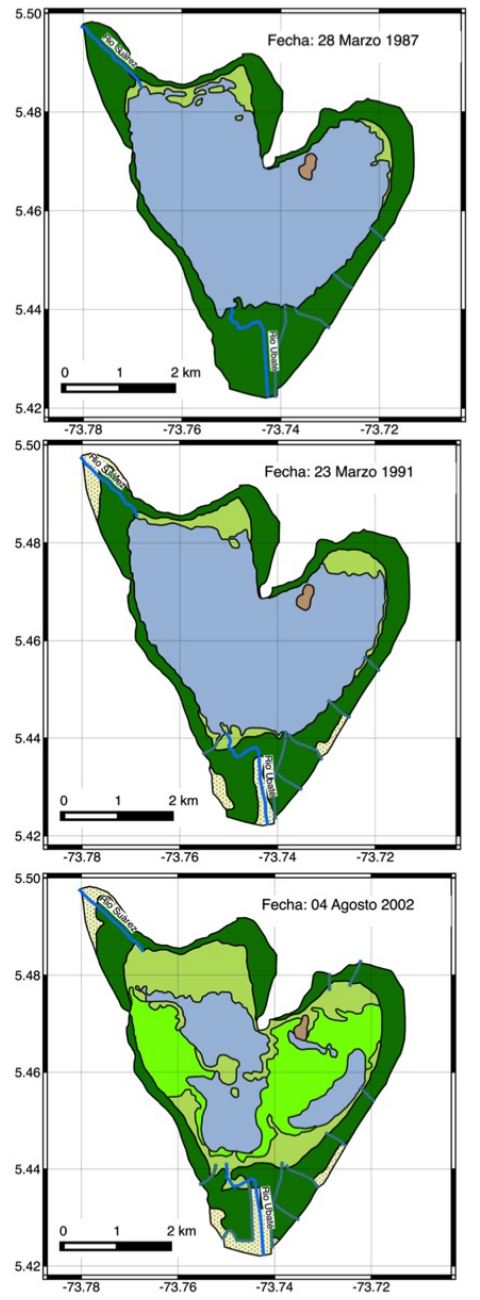

\begin{tabular}{|l|}
\hline Leyenda \\
Coberturas \\
\hline Vegetación acuática flotante \\
Areas cultivadas \\
Vegetación emergente de ribera \\
Espejo de agua \\
Isla \\
\hline Praderas emergentes \\
Canales \\
\hline Rios
\end{tabular}


Figura 2. Dinámica temporal de porcentajes de ocupación de las coberturas vegetales acuáticas y del espejo de agua de la laguna de Fúquene correspondiente al periodo $1984-2003$

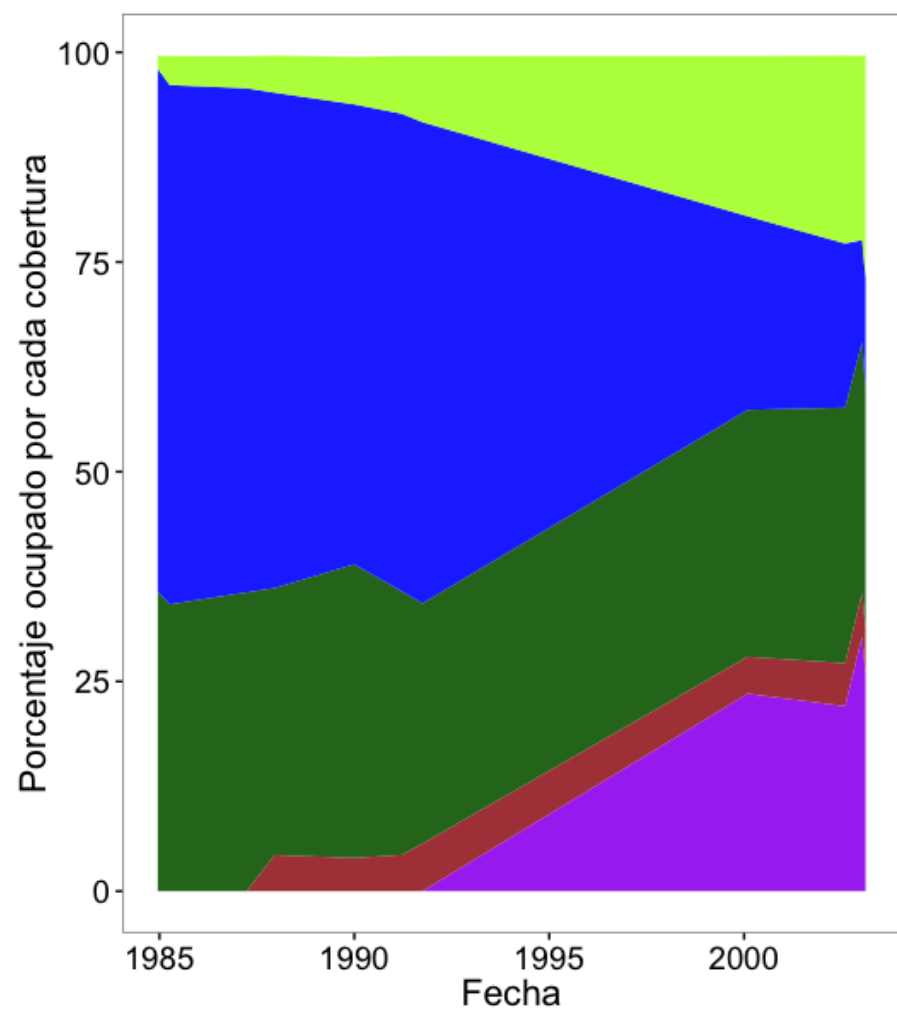

\section{Coberturas \\ Áreas cultivadas}

Espejo de agua

Praderas emergentes

Vegetación acuática flotante

Vegetación emergente de ribera

Figura 3. Representación gráfica de la tabla de correlaciones lineales de Spearman correspondiente a las coberturas acuáticas vegetales y al espejo de agua de la laguna de Fúquene

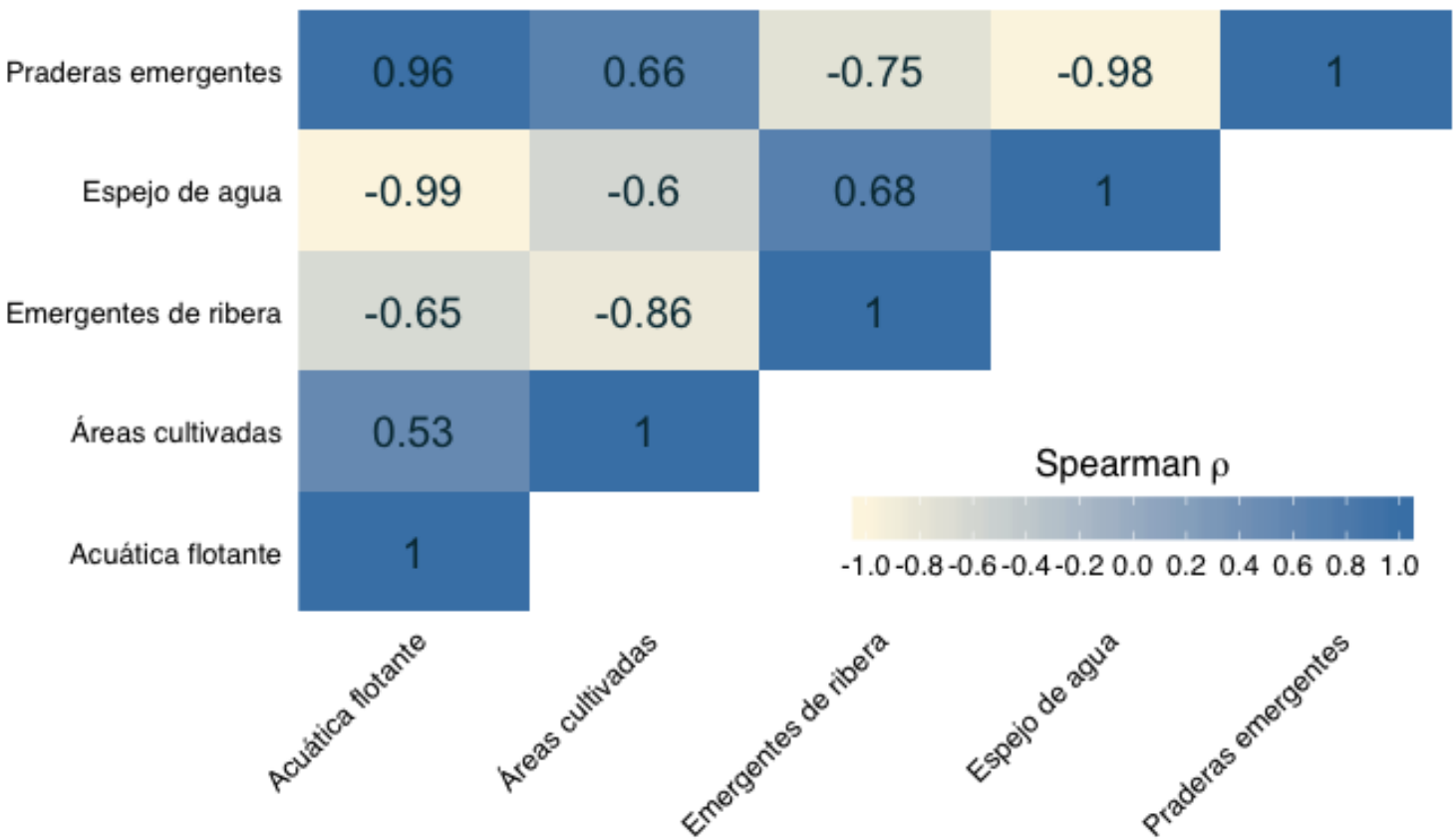


Figura 4. Regresión lineal del área del espejo de agua de la laguna de Fúquene en función del tiempo. La figura incluye los valores observados empleados en la regresión, así como el intervalo de confianza de la regresión

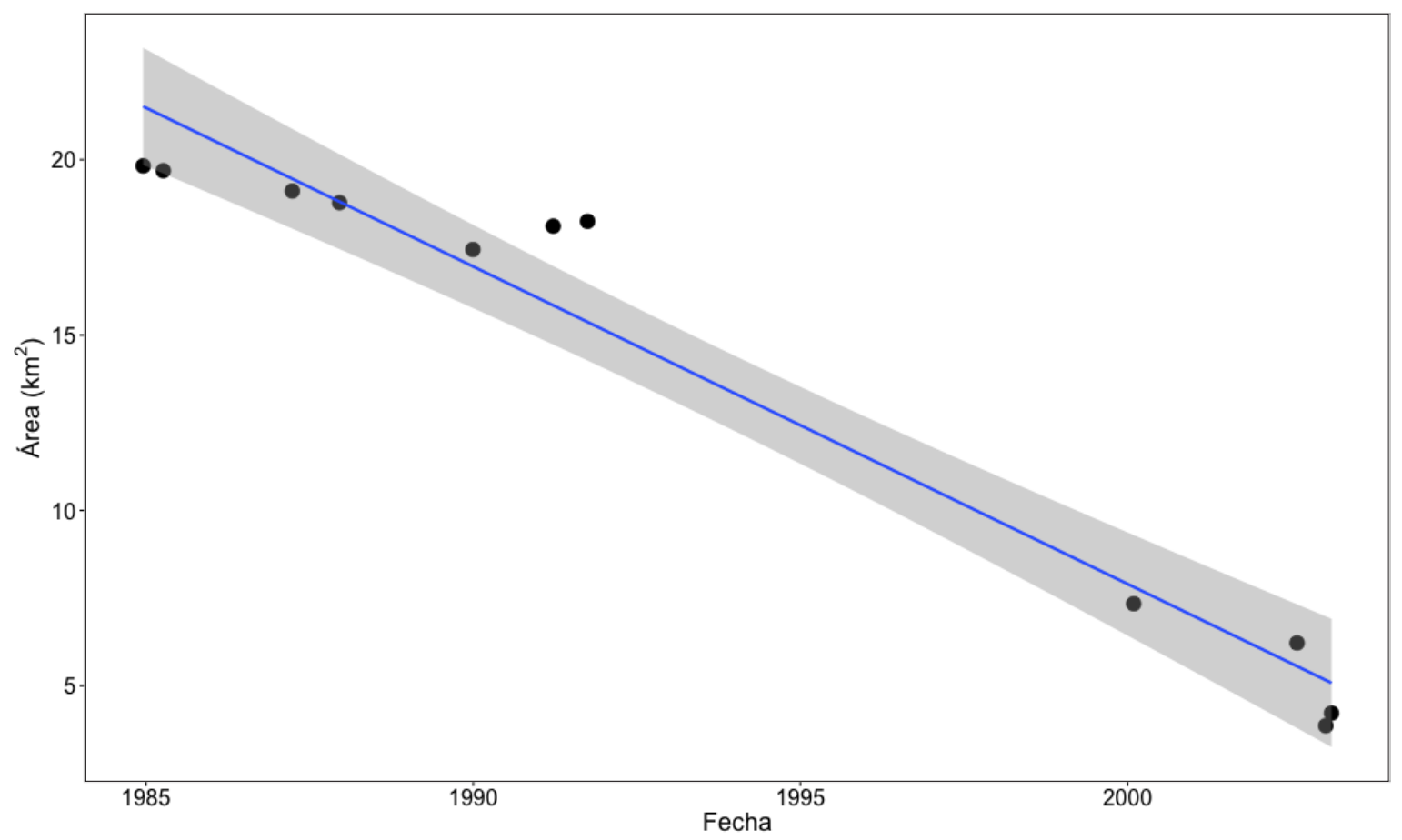

para confirmar los patrones de crecimiento de las diferentes coberturas; no obstante, es necesario que se analicen en función de la información disponible. Las imágenes analizadas presentan un vacío de información del año 1991 al 2000, en una ventana de tiempo total de aproximadamente veinte años. Esto hace que en la parte central de la ventana de tiempo no se tenga información del comportamiento de las coberturas, de tal forma que se presentan cambios abruptos en los resultados entre la última imagen analizada, de 1991, y la del año 2000. Esta condición favorece el aumento de los valores de correlación, ya sea en sentido positivo o negativo, dependiendo del caso. Si fuera posible contar con información de mayor resolución espacial podrían establecerse de manera más adecuada las tendencias de crecimiento tanto de las coberturas como del espejo de agua.

El resultado del análisis de regresión del área del espejo del agua en función del tiempo se presenta en la figura 4, donde puede confirmarse la disminución progresiva del espejo de agua en el periodo de tiempo considerado. El valor de la pendiente obtenido en el modelo fue de -0.88 , valor que indica que cada año la laguna ha venido perdiendo $0.88 \mathrm{~km}^{2}$ de espejo de agua. Si en la actualidad, a partir de los datos obtenidos en los años anteriores, se realizara una prospección del área del espejo de agua que en este momento debería existir, daría como resultado que ya se habría perdido en su totalidad dicha cobertura. Sin embargo, diferentes factores, como la ola invernal causada por el fenómeno de La Niña, así como las acciones que se han emprendido en el último tiempo para recuperar la laguna, han impedido que esto ocurra.

El trabajo de Bustamante (2010) predijo que en el año 2035 desaparecería el espejo de agua como consecuencia del crecimiento del buchón de agua. A pesar de las limitaciones espaciales y temporales del modelo calibrado en este trabajo, así como la tendencia que se presenta a partir de la interpretación de las imágenes satelitales del presente, es clara la necesidad de evaluar las acciones correctivas que se han venido tomando en torno a la recuperación de la laguna de Fúquene, con el fin de evitar que efectivamente se llegue a la desaparición del espejo de agua, tal como ocurrió con la laguna de Palacio, que ya no es navegable, y cuyos peces murieron por desoxigenación (CAR, 2000).

Entre las causas que han favorecido la alteración de los patrones de regulación de agua están los distritos de 
riego y drenaje. En la laguna de Fúquene ha sido notorio el efecto que ha tenido la construcción de drenajes desde zonas agrícolas hacia la laguna. Esto, asociado a antiguas políticas de desecación, ha ocasionado que Fúquene sea una laguna somera o panda, y esto incrementa su vulnerabilidad frente a fenómenos con la descarga de sedimentos y alteraciones climáticas (Rodríguez-Briceño, 2012). Si la laguna tuviera un mayor espejo de agua y mayor profundidad, y se disminuyera el aporte de sedimentos, el cuerpo de agua podría amortiguar cambios, como el incremento de la temperatura, lo cual favorecería su recuperación (Vidal, Delgado \& Andrade, 2012).

Las variaciones en los tipos de coberturas en la laguna traen consigo variaciones en las comunidades de otros organismos. Clavijo-Awazacko \& Amarillo-Suárez (2013) caracterizaron y compararon la diversidad de artrópodos terrestres asociada a las comunidades vegetales de algunos humedales de Bogotá. En los resultados de ese trabajo se sugiere la existencia de una relación entre el grado de perturbación de cada humedal (en términos de las comunidades vegetales presentes) y la diversidad y estructura de las comunidades de insectos (Clavijo-Awazacko \& Amarillo-Suárez, 2013).

Adicionalmente, los cambios en las coberturas vegetales acuáticas no son la única evidencia de la perturbación de la biodiversidad en la laguna. En el caso específico de Fúquene ya se ha documentado una disminución en las poblaciones de Eremophilus mutisii (capitán de la sabana) debido a procesos de eutrofización de la laguna (Rodríguez et al. 2005; Espitia-Contreras, 2013). Igualmente, se han observado lesiones en esta especie, como úlceras cutáneas asociadas a bacterias Pseudomonas spp. y Escherichia coli (Rodríguez, Montaño, Rodríguez, 2005), que son comunes en heces fecales animales como las descargadas a la laguna.

Por esta razón, aunque en los últimos años se ha trabajado en la recuperación y conservación de la laguna, usando como principal estrategia el dragado de sedimentos, si no se corrigen problemas como la descarga de sedimentos desde las fuentes al río Ubaté y a los canales que desembocan en la laguna, estos terminarán siendo esfuerzos aislados que no tendrán un efecto significativo para solucionar el problema.

\section{CONCLUSIONES}

La laguna de Fúquene presentó un proceso drástico de pérdida de su espejo de agua durante el periodo comprendido entre 1984 y 2003: pasó de tener 19.8 $\mathrm{km}^{2}$ de espejo de agua a solo $4.2 \mathrm{~km}^{2}$. El espejo de agua ha sido reemplazado por vegetación acuática. Algunas áreas en las orillas de la laguna han sido desecadas e incorporadas a actividades agrícolas.

Los procesos de enriquecimiento de nutrientes, descarga de materia orgánica y sedimentos se ven reflejados en la aparición y expansión de diferentes tipos de coberturas acuáticas vegetales, como las flotantes y las emergentes. La presencia de estas coberturas fue claramente identificada en las imágenes de satélite consideradas en el presente estudio.

Las principales causas asociadas a las variaciones observadas en el espejo de agua y las coberturas vegetales acuáticas son la descarga de sedimentos orgánicos e inorgánicos desde los centros poblados y áreas agrícolas aguas arriba de la laguna. También, aunque en menor medida, la desecación de áreas de la laguna para ser aprovechadas con fines agrícolas. Las principales consecuencias relacionadas son la alteración de comunidades vegetales y animales, específicamente, la expansión de plantas acuáticas en el área de la laguna y la modificación de las comunidades de animales asociadas a este tipo de vegetación.

La disponibilidad restringida de imágenes de satélite no permitió establecer la tendencia actual de las áreas del espejo de agua y de las coberturas vegetales. El análisis realizado hasta el año 2003 no permite evaluar el efecto de las estrategias de restauración y conservación de la laguna que se han puesto en marcha durante los últimos años. El acceso a estas imágenes depende de la oferta que liberen los proveedores de dichas imágenes y de contar con los recursos necesarios para adquirirlas, en caso de que las imágenes tengan algún costo.

Aun cuando la desecación de la laguna y la pérdida del espejo de agua es un fenómeno plenamente descrito y comprendido en el caso de la laguna de Fúquene, el uso de herramientas de sensorización remota y de sistemas de información geográfica permitió una cuantificación bastante precisa de los cambios espaciales y temporales que han presentado 
las coberturas de la laguna a lo largo del periodo de tiempo considerado.

La técnica de clasificación de coberturas empleada en el presente trabajo y la interpretación visual de imágenes fueron suficientes para realizar la discriminación de las coberturas incluidas. Las imágenes a falso color construidas a partir de la información contenida en las bandas de las imágenes satelitales presentaron el suficiente contraste para que la interpretación visual fuera adecuada con miras a una correcta clasificación.

\section{REFERENCIAS}

Aksoy, E. \& Özsoy, G. (2002). Investigation of multitemporal land use/cover and shoreline changes of the Uluabat Lake Ramsar site using RS and GIS. International Conference in Sustainable Land Use and Management, Çanakkale, Turkey.

Alberti, M., Booth, D., Hill, K., Coburn, B., Avolio, C., Coe, S. \& Spirandelli, D. (2007). The impact of urban patterns on aquatic ecosystems: An empirical analysis in Puget lowland sub-basins. Landscape and Urban Planning, 80, 345-361.

Alexandridis, T. K., Takavakoglou, V., Crisman, T. L. \& Zalidis, G. C. (2007). Remote sensing and GIS techniques for selecting a sustainable scenario for Lake Koronia, Greece. Environmental Management, 39, 278-290.

Alsdorf, D. E., Rodríguez, E. \& Lettenmaier, D. P. (2007). Measuring surface water from space. Reviews of Geophysics, 45(2), doi: http://dx.doi. org/10.1029/2006RG000197

Bastawesy, M. A., Khalaf, F. I., \& Arafat, S. M. (2008). The use of remote sensing and GIS for the estimation of water loss from Tushka lakes, southwestern desert, Egypt. Journal of African Earth Science, 52, 73-80.

Bustamante, S. (2010). Modelado de especies invasoras, caso de estudio: pérdida del espejo de agua en la laguna de Fúquene por invasión del buchón (Eichornia crassipes) (tesis de maestría). Pontificia Universidad Javeriana, Bogotá, Colombia.
Camargo, J. A., \& Alonso, A. (2006). Ecological and toxicological effects of inorganic nitrogen pollution in aquatic ecosystems: A global assessment. Environment International, 32, 831-849.

CAR (Corporación Autónoma Regional de Cundinamarca). (2000). Fúquene: el lecho de la zorra. Corporación Autónoma Regional de Cundinamarca, Bogotá, Colombia.

Clavijo-Awazacko, H. \& Amarillo-Suárez, Á. (2013). Variación taxonómica y funcional en la artropofauna asociada a comunidades vegetales en humedales altoandinos (Colombia). Revista Colombiana de Entomología, 39(1), 155.

Espitia-Contreras, J. P. (2013). Análisis de la aplicación del enfoque ecosistémico en la estrategia de manejo ambiental de la cuenca Ubaté-Suárez implementada en la laguna de Fúquene durante el período 2007-2009 (tesis de pregrado). Universidad Militar Nueva Granada, Bogotá, Colombia.

Giardino, C., Bresciani, M., Villa, P. \& Martinelli, A. (2010). Application of remote sensing in water resource management: The case study of Lake Trasimeno, Italy. Water Resources Management, 24, 3885-3899.

GRASS Development Team (2012). Geographic resources analysis support system (GRASS 6) programmer's manual. Open Source Geospatial Foundation Project.

Hernández-Cornejo, R., Koedam, N., Ruiz-Luna, A., Troell, M. \& Dahdouh-Guebas, F. (2005). Remote sensing and ethnobotanical assessment of the mangrove forest changes in the NavachisteSan Ignacio-Macapule lagoon complex, Sinaloa, Mexico. Ecology and Society, 10(1), 16.

Khan, F. A., Naushin, F., Rehman, F., Masoodi, A., Irfan, M., Hashmi, F., \& Ansari, A. A. (2014). Eutrophication: Global scenario and local threat to dynamics of aquatic ecosystems. En A. A. Ansari y S. S. Gill, Sarvajeet (eds.), Eutrophication: Causes, consequences and control, pp. 17-27. Springer Netherlands.

Melo, L. H. \& Camacho, M. A. (2005). Interpretación visual de imágenes de sensores remotos y su 
aplicación en levantamientos de cobertura física y uso de la tierra. Bogotá: IGAC.

Navarro-Ortega, A., Acuña, V., Bellin, A., Burek, P., Cassiani, G., Choukr-Allah, R., Dolédec, S., Elosegi, A., Ferrari, F., Ginebreda, A., \& Grathwohl, P. (2015). Managing the effects of multiple stressors on aquatic ecosystems under water scarcity: The Globaqua Project. Science of the Total Environment, 503, 3-9.

Omar, W. A., Zaghloul, K. H., Abdel-Khalek, A. A., \& Abo-Hegab, S. (2013). Risk assessment and toxic effects of metal pollution in two cultured and wild fish species from highly degraded aquatic habitats. Archives of Environmental Contamination and Toxicology, 65(4), 753-764.

Papastergiadou, E. S., Retalis, A., Apostolakis, A., \& Georgiadis, Th. (2008). Environmental monitoring of spatio-temporal changes using remote sensing and GIS in a Mediterranean wetland of Northern Greece. Water Resources Management, 22, 579594.

Parra, A., \& Hernández, F. L. (2010). Identificación y delimitación de humedales lénticos en el valle alto del río Cauca mediante el procesamiento digital de imágenes de satélite. Ingeniería de Recursos Naturales y del Ambiente, 9, 77-88.

Pathirana, S., Perera, K., \& Sanaa, H. (2015). Impact of climate change on water resources in MENA countries: An assessment of temporal changes of land cover/land use and water resources using multi-temporal MODIS and Landsat data and GIS techniques. En Proceedings of the International Symposium on Remote Sensing (ISRS 2015). University of Southern, Queensland.

Pernett, X. (2006). Proceso para cuantificar e interpretar las características físicas y químicas del suelo que reglan su función en relación con el agua, el almacenamiento de nutrientes y la infiltración (caso de la cuenca de la laguna de Fúquene). Lima: Centro Internacional de la Papa, LuzAzul Gráfica.

QGIS Development Team (2009). QGIS Geographic Information System. Open Source Geospatial Foundation. Disponible en: http://qgis.osgeo.org
R Core Team. (2014). R: A language and environment for statistical computing. $R$ Foundation for Statistical Computing, Vienna, Austria. Disponible en: http://www.R-project.org/

Rondón, G. A. \& Vergara, K. V. (2012). Estudio espacialtemporal 1962-2011 de las lagunas Sayhuacocha, Raurac, Ampatococha y Tutayoc, Huancavelica, Perú. Revista Geográfica, 151, 151-177.

Rodríguez-Briceño, L. S. (2012). Plan de Desarrollo municipio de Fúquene 2012-2015. Fúquene: Concejo Municipal de Fúquene.

Rodríguez, A., Montaño, E., \& Rodríguez, J. (2005). Evaluación preliminar de las patologías cutáneas asociadas con la aclimatación en cautiverio del "capitán de la sabana", Eremophilus mutisii. Acta Biológica Colombiana, 10(2), 123-128.

Sawaya, K. E., Olmanson, L. G., Heinert, N. J., Brezonik, P. L., \& Bauer, M. E. (2003). Extending satellite remote sensing to local scales: Land and water resource monitoring using high-resolution imagery. Remote Sensing of Environment, 88(1), pp. 144-156.

Sexton, J. O., Urban, D. L., Donohue, M. J., \& Song, C. (2013). Long-term land cover dynamics by multitemporal classification across the Landsat- 5 record. Remote Sensing of Environment, 128, 246-258.

Tarasenko, T. V. (2013). Interannual variations in the areas of thermokarst lakes in Central Yakutia. Water Resources, 40, 111-119.

Vidal, L.F., Delgado, J. \& Andrade, G.I. (2012). Laguna de Fúquene: entender la crisis, visualizar el futuro $y$ acordar el camino. Gestión de Humedales 2: 17 $-26$.

Vidal, L. F. \& Pérez, G. I. A. (2007). Fúquene, Cucunubá y Palacio: conservación de la biodiversidad y manejo sostenible de un ecosistema lagunar andino. Bogotá: Instituto de Investigación de Recursos Biológicos Alexander von Humboldt.

Were, K. O., Dick, $\varnothing$. B. \& Singh, B. R. (2013). Remotely sensing the spatial and temporal land cover changes in Eastern Mau forest reserve and Lake Nakuru drainage basin, Kenya. Applied Geography, $41,75-86$. 
Woodward, G., Gessner, M. O., Giller, P. S., Gulis, V., Hladyz, S., Lecerf, A., Malmqvist, B., McKie, B. G., Tiegs, S. D., Cariss, H., \& Dobson, M. (2012). Continental-scale effects of nutrient pollution on stream ecosystem functioning. Science, 336(6087), 1438-1440. 